\title{
Qualidade de vida dos profissionais de enfermagem que conciliam trabalho e estudo em um centro universitário privado na cidade de Recife/PE
}

\author{
Quality of life of nursing professionals who combine work and study in a private university center in \\ the city of Recife/PE
}

Tássia Rayane de Arruda*, Wagner Ferreira dos Santos, Natália Izabel Domingos de Freitas

${ }^{1}$ Graduada em Enfermagem, Recife, Pernambuco, Brasil. *Autor para correspondência. E-mail: rayannearruda2@hotmail.com

Resumo: Introdução: A qualidade de vida de acordo com a Organização Mundial de Saúde (OMS) é uma percepção do indivíduo quando a sua posição de vida baseado em sua cultura e dos seus sistemas de valores no qual está inserida, em relação aos seus objetivos, padrões de vida e preocupações. Objetivo foi avaliar os fatores que interferem na qualidade de vida dos profissionais de enfermagem que conciliam trabalho e estudo no Centro Universitário Estácio do Recife/PE. Caracterizar o perfil socioeconômico destes acadêmicos de enfermagem e identificar os fatores estressores que interferem na qualidade de vida deles. Material e métodos: Foi realizado um estudo descritivo exploratório de corte transversal com abordagem quantitativa no Centro Universitário Estácio do Recife-PE. A população foi composta por acadêmicos do curso de Graduação em enfermagem devidamente matriculados no $6^{\circ}$ e $7^{\circ}$ Períodos manhã e noite. No período de outubro e novembro de 20017. Utilizou-se como instrumentos dois questionários, um de dados demográficos e o outro de Avaliação da QV o WHOQOL-Bref. Resultados e discussão: A caracterização social que a maioria da amostra apresentou foi: sexo feminino, faixa etária era 32 a 37 anos; solteiros; possuíam dois filhos; residiam na capital; estudavam de 03 a 04 dias, com carga horária entre 10 a15 horas semanais; 01 vínculo empregatício de 40 horas semanais e regime de $12 \times 36$. Com relação ao estudo do instrumento WHOQUOL-Bref, que proporcionou através dos resultados, respostas significantes, como por exemplo: satisfação com qualidade de vida, condições de moradia; capacidade de energia e em contrapartida preocupantes, como exemplos: insatisfação financeira, falta de tratamento médico, entre outros. Considerações finais: Os resultados encontrados demonstram a importância de se implantar estratégias que busquem melhorar ainda mais a qualidade de vida destes profissionais de enfermagem nos âmbitos físico, social, ambiental e psicológico.

Palavras-chave: qualidade de vida, instrumentos de medida, promoção de saúde.

\begin{abstract}
Introduction: A quality of life according to the World Health Organization (WHO) is a perception of the individual when his position of life in his life and his value system without which he is inserted, in relation to his objectives, of life and concerns. The objective was to evaluate the factors that interfere in the quality of life of nursing professionals that reconcile work and study. Centro Universitário Estácio do Recife / PE. Characterize the socioeconomic profile of these nursing students and identify the stressors that interfere in quality of life. Material and methods: A descriptive exploratory cross-sectional study with a quantitative approach was carried out at Estácio University Center in Recife-PE. The population was composed of graduate undergraduate nursing students duly enrolled in the 6th and 7th Periods of morning and night. In the period of October and November of 20017. Two questionnaires were used as instruments, one of the demographic data and the other of the QOL Assessment, WHOQOL-Bref. Results and discussion: Social characterization of an edition of the presented sample: female, age group of 32 to 37 years; singles; they had two children; they resided in the capital; studied from 03 to 04 days, with a workload of 10 to 15 hours per week; 01 work contract of 40 hours per week and 12x36 regime. Regarding the study of the WHOQUOL-Bref instrument, which provides, through the results, significant answers, such as: satisfaction with quality of life, living conditions; energy capacity and, on the other hand, worrying, such as: financial dissatisfaction, lack of medical treatment, among others. Final considerations: The results show the importance of implementing a strategy that aims to improve the quality of life of these nursing professionals in the physical, social, environmental and psychological domains.
\end{abstract}

Keywords: quality of life, measuring instruments, health promotion. 


\section{Introdução}

Schrader et al. (2012) afirmam que a qualidade de vida de acordo com a Organização Mundial da Saúde, (OMS) é uma percepção do indivíduo quando a sua posição de vida baseado em sua cultura e dos seus sistemas de valores nos quais está inserido, em relação aos seus objetivos, padrões de vida e preocupações. Desse modo, a qualidade de vida pode ser alcançada a partir da satisfação e realização profissional e social. Diante disso o trabalho configura um elemento central. É por meio dele que as pessoas se satisfazem. Por estar conectada aos aspectos de saúde por diversas vezes, a qualidade de vida é usada como sinônimo de saúde; apesar da estreita relação entre as duas condições pode-se afirmar que qualidade de vida é ter saúde.

A qualidade de vida no trabalho é uma compreensão abrangente das condições de vida no ambiente laboral, que incluem aspectos de bem-estar, saúde, segurança física, e a capacidade dos profissionais de realizar as tarefas com segurança. É importante intervir no ambiente de trabalho para amenizar as dificuldades nas atividades e melhorar a saúde dos profissionais. Assim, como objetivo identificar e analisar a produção de conhecimento sobre ambiente de a qualidade de vida no trabalho QVT (Hipólito et al., 2017).

Em decorrência da situação socioeconômica capitalista em que vivemos atualmente muitas pessoas precisam trabalhar e estudar para se manter, a fim de pagar os cursos, trabalhadores dispostos a aperfeiçoamentos obrigam-se a trabalhar a noite e estudar durante o dia. A escolha pelo trabalho noturno envolve vários motivos, entre eles; a remuneração diferenciada e a possível, conciliação entre trabalho e estudo. Nesse sentido entende-se que a qualidade de vida de quem trabalha e estuda está ameaçada (Fontana \& Brigo, 2012).

Os processos de trabalho estão cada vez mais complexos e exigentes, carga horária excessiva, bem como; as repercussões que estão entrelaçadas na vida familiar e social, aliadas as dificuldades financeiras têm acarretado um determinado comprometimento sério a qualidade de vida dos profissionais de enfermagem (Marcitelli, 2011).

Os profissionais de enfermagem além de enfrentarem situações desafiadoras em circunstancias laborais, com o manejo dos pacientes graves e muitas vezes de seus familiares, A dor, cansaço, danos à audição, desordens no sono dentre outros fatores, tiveram grande repercussão na saúde do trabalhador de enfermagem (Bordignon \& Monteiro, 2016).

Os fatores de exposição das cargas de trabalho levam a processos de desgaste físico-psíquico que permitem traçar um perfil de agravos aos profissionais de enfermagem de modo a interferir na assistência prestada ao cliente. Muito se fala na qualidade dos serviços prestados ao paciente e acabam se esquecendo de manter a qualidade de vida dos profissionais atuantes. Com ênfase aos profissionais que podem ser acometidos por desgastes físicos e emocionais são os técnicos e os auxiliares de enfermagem, que são os mais propícios a esses danos à saúde pois prestam os cuidados diretos a pacientes em ambientes muitas vezes insalubres, turnos alternados, aumento de esforços físicos e principalmente a exposição a agentes biológicos (Freire \& Costa, 2016).

O trabalho torna o indivíduo um ser produtivo, na medida em que contribui para a criação de elementos necessários a sobrevivência humana. Ele insere o ser humano na sociedade; e é de extrema responsabilidade social de toda empresa adotar medidas para um trabalho saudável e em ambientes apropriados (Leal et al., 2013).

O monitoramento de saúde do trabalhador é uma ferramenta indispensável para mudar essa realidade, permitindo monitorar as cargas de trabalho envolvidas no processo de adoecimento dos mesmos; e a caracterização do perfil do trabalhador (Santana et al., 2016).

Considera-se que a qualidade de vida vem sendo avaliada nas diversas modalidades, principalmente nos profissionais de enfermagem, e tornam-se mais relevantes devido à complexidade existente na vida dos trabalhadores que enfrentam situações que acabam deixando-os expostos a riscos que envolvem a profissão, dentre eles estão presentes: as cargas excessivas de trabalho, remuneração inadequada e muitas vezes ambiente insalubre, desgastes físicos mentais, desfavorecendo a qualidade de vida (Gomes \& Coqueiro, 2016). Fatores que interferem na qualidade de vida dos profissionais de enfermagem são acentuados a esses profissionais quando eles têm que reservar momentos para conciliar trabalho e estudo. Diante disso, questiona-se: A qualidade de vida dos profissionais de enfermagem é afetada ao conciliar trabalho e estudo?

\section{Revisão}

$\mathrm{O}$ interesse pelo tema qualidade de vida tem aumentado nas últimas décadas, no entanto, ainda não podemos defini-la em sua totalidade uma vez que envolve aspectos individuais de cada pessoa. Qualidade de vida é empregada como sinônimo de saúde por estarem conectada a seus aspectos. Afirmar que qualidade de 
vida é ter saúde é reduzi-la a um único fragmento de bem-estar humano. Sabendo que a população de estudantes que conciliam trabalho e estudo vem crescendo ao longo dos anos ao refletir sobre os aspectos relacionados a carga horária extensa em sala de aula, lazer reduzido ansiedade para organizar seu tempo de estudo e trabalho e muitas vezes a angustia pelo baixo desempenho acadêmico nota-se que a qualidade de vida se torna prejudicada (Moura et al., 2016).

A qualidade de vida não está atrelada apenas a um único aspecto de vida do homem mais sim a diversos fatores que determinam que o indivíduo possa estar em harmonia bio, pisco, sócio, cultural isso envolve saúde mental, relação no trabalho entre outros. Segundo a Organização Mundial de Saúde (OMS) o termo qualidade de vida é definido como a percepção do indivíduo de sua posição na vida no contexto de cultura e sistema de valores nos quais ele vive e em relação a seus objetivos, expectativas padrões e preocupações (Santos Fonseca et al., 2014).

Qualidade de vida encerra escolhas de bem-estar e percepção do que pode ser feito para atender expectativas de gestores e trabalhadores. $\mathrm{O}$ stress e a insatisfação profissional estão presentes no cotidiano dos profissionais de enfermagem, e estão relacionados a diversos fatores entre eles estão: ambiente, duração de jornada de trabalho, grau elevado de exigência quanto as responsabilidades (Zavala et al., 2016).

Um dos grandes eixos da concepção de qualidade de vida no trabalho é a concepção de bem-estar do empregado o Bem-estar não apenas no sentido psicológico, como eixo da qualidade de vida no trabalho está atrelada a duas linhas metodológicas. Uma linha fatorial que aborda o conceito da promoção da saúde, a segunda linha de estudo se divide em alguns critérios como: a significância do trabalho, a orientação do sujeito e a coerência entre o sujeito e o trabalho (Sampaio, 2012).

Considerando a centralidade do trabalho na vida humana a organização internacional do trabalho (OIT) e a organização mundial de saúde (OMS) assume um estreito relacionamento entre o trabalho e saúde, admitindo-se por sua vez uma considerável influência do primeiro termo da saúde do trabalhador e na construção da sua subjetividade. São múltiplos os estudos que consensualmente reconhecem a relação entre a organização e o mal-estar no trabalho (Mendonça \& Araújo, 2016).

O trabalho está centrado no processo de humanização e autonomia dos indivíduos, classificam-se numa categoria intermediaria fornecendo o salto ontológico das formas pré-humanas para o ser social. As relações entre os fatores de exposição das cargas de trabalho ocasionam os processos de desgaste físico psíquico que permitem traçar um perfil característico de agravos aos profissionais de enfermagem de modo a interferir na qualidade do atendimento ao cliente (Freire \& Costa, 2016).

Para compor o contexto da qualidade de vida o trabalho é entendido como parte integrante na vida em uma sociedade produtiva é um fator imprescindível para a satisfação pessoal sendo um meio onde o indivíduo consegue sua identidade e reconhecimento social. Assim o trabalho passou a ocupar um lugar central na vida do homem e depende de como ele é executado pode ter fins lucrativos ou pode gerar desgastes físicos e psíquicos ao profissional (Silva et al., 2015).

O trabalho está presente na vida do homem ao longo da história da humanidade. Historicamente, homem e trabalho se interligam de diferentes maneiras, conforme a sociedade e o período ao qual nos referimos, de modo que os significados sociais atribuídos ao trabalho sempre têm influenciado o contexto vivenciado pelo homem. Trabalho pode ser entendido como fonte de prazer e realização; representa o que de mais humano existe no homem, a capacidade de expressar sua "marca essencial" - a subjetividade -, fator fundamental ao equilíbrio e desenvolvimento humano (Cavalheiro \& Tolfo, 2011).

O mundo contemporâneo e as novas exigências do mercado exigem profissionais cada vez mais especializados e capacitados. Para atender a essas exigências, os profissionais precisam desdobrar-se desenvolvendo várias atividades diárias, mas o dinamismo e a inconstância do mercado acabam gerando tensões, medo e estresse, afetando a qualidade de vida, as tarefas diárias e a interação com as pessoas no ambiente de trabalho (Lima et al., 2015).

Várias abordagens têm sido desenvolvidas para avaliar o impacto do estresse no trabalho sobre a saúde entre elas a modelo demanda controle, enfatizando que o desequilíbrio entre a demanda psicológica e o controle sobre o processo produtivo resulta em desgaste, perda de habilidades e do interesse, afetando a saúde do trabalhador (Costa et al., 2017).

O stress é caracterizado como um processo psicofisiológico com respostas que envolvem o sistema nervoso autônomo e o sistema endócrino. Pode verificar também os sintomas de irregularidade hormonal que leva ao agravamento da saúde do indivíduo. A exposição prolongada aos agentes estressores implica no baixo desempenho profissional, baixa autoestima, prejudicando todo o trabalho realizado. Pode-se verificar que o stress se tornou um termo comum nos dias atuais e estar presente principalmente no cotidiano dos profissionais de enfermagem (Ratochinski et al., 2016). 
A qualidade de vida dos profissionais de saúde entre técnicos de enfermagem e enfermeiros cujas atribuições são especificas e que a saúde desses profissionais necessita de cuidados. A maioria das vezes esses profissionais são expostos a má qualidade de vida, e de grande importância que as empresas avaliem a qualidade de vida dos mesmos, pois eles estão, boa parte do tempo com indivíduos que necessitam de cuidado (Rios et al., 2010).

De modo geral, não está estabelecida um consenso cobre o conceito e qual seria a melhor forma de avaliar a qualidade de vida no trabalho e ou satisfação dos trabalhadores da enfermagem dentre os estudos realizados damos ênfase maior na dimensão patológica, inclusive no processo de adoecimento e fatores de risco para os profissionais da saúde ligados a carga biológica, física, e química a que são submetidos especialmente no ambiente hospitalar (Daubermann \& Tonete, 2012).

Cotidianamente, verifica-se o quanto a vivência de rotinas exaustivas, estresse, precariedade de cuidados de enfermagem, falta de diálogo, banalização da morte e burocracia, entre outras acompanhadas de sentimento de impotência frente às situações de aparente descaso em relação aos pacientes, influencia a forma de ser e fazer dos trabalhadores de enfermagem, o que lhes pode provocar desconforto e sofrimento, sem comumente identificá-lo como sofrimento moral (Barlem et al., 2013).

Inserida nesse contexto a profissão de enfermagem se destaca, tendo em vista o grande número de profissionais no mercado de trabalho. O elevado custo de vida obriga os enfermeiros a exercer uma jornada excessiva de trabalho, fazendo com que haja uma grande exposição desses profissionais aos possíveis agentes causadores da má qualidade de vida, e consequentemente comprometendo a qualidade da assistência prestada (Acioli Neto et al., 2013).

Os profissionais de enfermagem atuam em condições que há muito tempo são consideradas inadequadas devido às condições do ambiente e as atividades insalubres por estes executados. $O$ desprestigio social são fatores associados as condições de trabalho do enfermeiro, que vem refletindo na qualidade da assistência prestada, levando ao abandono da profissão e consequentemente a falta de profissionais no mercado de trabalho (Amaral et al., 2015).

Entre os trabalhadores da área de saúde a relação qualidade de vida e trabalho é ainda maior devido às dificuldades encontradas no exercício profissional, as quais abrangem um ambiente formado por intensos estímulos emocionais, como o contato com a dor e o sofrimento, o lidar com pacientes terminais, deprimidos, queixosos, rebeldes e não aderentes ao tratamento, e o lidar com as limitações do sistema assistencial que se contrapõem às demandas e expectativas dos pacientes e familiares (Mascarenhas et al., 2013).

Entre os trabalhadores da saúde, os profissionais de enfermagem estão no grupo dos mais propensos aos problemas de saúde mental, dentre os quais a depressão e o risco de suicídio, porque lidam com o sofrimento humano, a dor, a alegria, tristeza e necessitam ofertar ajuda àqueles que necessitam de seus cuidados. Destacam-se, ainda, outros fatores comumente encontrados, como as condições difíceis de trabalho e a falta de reconhecimento profissional. Sabe-se que a depressão é uma das três doenças mais referidas pelos trabalhadores de enfermagem, para tanto, os responsáveis pelos serviços de saúde devem identificar este problema precocemente, promover a saúde no trabalho, evitar desfechos tristes e fatais, bem como a diminuição ou perda da qualidade da assistência prestada (Silva et al., 2016).

Maciel e Oliveira (2014) afirmam que a enfermagem é uma profissão que zela pela saúde e bem-estar do paciente e da sua coletividade. No entanto, durante o seu exercício, os profissionais de enfermagem estão sujeitos a sofrerem grande estresse psicológico, principalmente aqueles que atuam no ambiente hospitalar, que é considerado o ambiente mais estressante dentre os estabelecimentos que prestam serviços de saúde.

O profissional de enfermagem passa por dificuldades no processo de trabalho, pois este o coloca frente a vários problemas que podem provocar estresse e consequentemente interfere na sua saúde e realização de atividades, pela falta de emprego muitos profissionais se sujeitam a adquirir mais de um emprego fazendo com que sua qualidade de vida fique prejudicada gerando fatores que implicam cada vez mais em sua saúde. (Silva \& Queiroz, 2011).

A equipe de enfermagem representa a maior força de trabalho presente nas instituições hospitalares, entretanto durante a realização da assistência existem limitações quanto ao número de trabalhadores e recursos materiais disponíveis, o que torna o trabalho desgastante, em vistas das cargas de trabalho presentes no ambiente, A exposição contínua às cargas de trabalho, a vivência diária de sentimentos de prazer e de sofrimento gera desgaste no trabalhador, e isto pode evoluir para o adoecimento. Desta forma, é necessário que o trabalhador compreenda as peculiaridades da profissão, e aproprie-se de estratégias que possam reduzir este desgaste profissional, buscando a preservação da sua saúde (Carvalho et al., 2017).

O trabalhar e estudar são atividades comuns aos indivíduos que ingressam na educação de nível médio, quanto superior nos dias de hoje. Os motivos que levam os estudantes a percorrerem este caminho é a 
melhoria das condições financeiras aliada com a realização profissional. O conceito trabalhador-estudante surgiu a partir do conceito de que este independe financeiramente e as vezes colaboram com o orçamento doméstico (Maier \& Mattos, 2016).

Entende-se por acadêmico ou estudante de enfermagem o ser humano que faz uma opção por cuidar e ajudar outros seres humanos a nascer e viver de forma saudável; a superar agravos à sua saúde; a conviver com limitações e encontrar um significado nessa experiência e; a morrerem com dignidade. No processo de preparar-se para realizar as várias ações que integram esse trabalho, com competência técnica, dialógica e política, enfrenta situações de sofrimento que podem contribuir tanto para processo de humanização deste trabalho, quanto para sua banalização. Apesar da percepção individual da Qualidade de Vida resultar da evolução histórica e social de cada ser humano, as considerações realizadas acerca dos Aspectos relacionados ao trabalho pactuam do mesmo ambiente físico e organizacional, o que possibilita a construção de um perfil de qualidade de vida para o grupo (Oliveira et al., 2011).

Fica evidente a importância do bem-estar e saúde do indivíduo no trabalho, pois é nele que se passa a maior parte do tempo. A qualidade de vida está diretamente relacionada com as necessidades e expectativas humanas e com a respectiva satisfação dessas, correspondendo ao bem-estar do indivíduo no ambiente de trabalho, expresso através de relações saudáveis e harmônicas. A qualidade de vida é fundamental para a execução de qualquer atividade, especialmente na enfermagem que tem um enfoque direcionado para a melhoria da qualidade de vida da população, tornando-se primordial que o profissional de enfermagem adquira plenas condições de trabalho e de vida (Santana et al., 2014).

Um dos grandes eixos da concepção de qualidade de vida no trabalho é a concepção de bem-estar do empregado o bem-estar não apenas no sentido psicológico, como eixo da qualidade de vida no trabalho está atrelada a duas linhas metodológicas. Uma linha fatorial que aborda o conceito da promoção da saúde, a segunda linha de estudo se divide em alguns critérios como: a significância do trabalho, a orientação do sujeito e a coerência entre o sujeito e o trabalho (Sampaio, 2012).

Qualidade de vida no trabalho é definida como a relação entre a satisfação do trabalhador com as atividades exercidas no ambiente de trabalho, atendendo suas necessidades e pretensões humanas, e o retorno que ele propicia para a empresa, promovendo seu sucesso perante a concorrência (Santana et al., 2014).

Várias abordagens têm sido desenvolvidas para avaliar o impacto do estresse no trabalho sobre a saúde entre elas a modelo demanda controle, enfatizando que o desequilíbrio entre a demanda psicológica e o controle sobre o processo produtivo resulta em desgaste, perda de habilidades e do interesse, afetando a saúde do trabalhador (Ueno et al., 2017).

A dimensão da exaustão emocional representa um grande problema em saúde, elas referem-se as sensações de que o trabalhador está no seu limite tanto físico, quanto emocional, se sentem fadigados, esgotados, as principais fontes de exaustão estão presentes na sobrecarga de trabalho e muitas vezes nos conflitos pessoais (Schmidt et al., 2013).

Entende-se que o contexto de qualidade de vida relacionado ao trabalho e entendido como parte importante na vida da sociedade, é através dele que conseguimos nossa realização satisfação pessoal, profissional e obtemos uma participação mais efetiva na sociedade. Assim o trabalho passou a ocupar um lugar importante na vida do homem dependendo de como vem sendo organizado pode interferir ou melhorar a qualidade de vida dos profissionais de enfermagem (Silva et al., 2016).

A precarização do trabalho em saúde vem sendo identificado como um dos obstáculos para o desenvolvimento do sistema de saúde público, comprometendo a relação entre os trabalhadores e o sistema de saúde, podendo prejudicar os serviços prestados pelo SUS, tanto no que diz respeito à qualidade quanto na continuidade dos mesmos. Com toda a importância e reconhecimento que parece ser atribuído ao trabalho do enfermeiro, a precarização do vínculo empregatício em saúde é uma realidade ainda presente na ESF e na conjuntura de trabalho deste profissional. A insuficiência na realização de concursos públicos e a deficiência no reconhecimento dos direitos trabalhistas fazem com que o vínculo laboral se torne fragilizadas (Viana et al., 2016).

\section{Materiais e métodos}

Foi realizado um estudo descritivo exploratório de corte transversal com abordagem quantitativa. A pesquisa descritiva descreve as características de determinada população ou fenômeno ou estabelecimento de relações entre variáveis, e a pesquisa exploratória visa à descoberta, o achado, a elucidação de fenômenos ou a explicação daqueles que não eram aceitos apesar de evidentes. A exploração representa, atualmente, um 
importante diferencial competitivo em termos de concorrência. A pesquisa quantitativa considera o que pode ser quantificável o que significa traduzir em números opiniões e informações para classificá-las e analisá-las (Lakatos \& Marconi, 2009). O local da pesquisa foi o Centro Universitário Estácio do Recife, no campus Abdias de Carvalho na região metropolitana da cidade do Recife, no Estado de Pernambuco. Composta com mais de 10.000 alunos regularmente matriculados, com 20 cursos entre presenciais e a distância. Todavia, o curso de enfermagem contém 1.200 alunos, com a carga horária 4.301 hora/aula. A população foi composta por 128 acadêmicos do curso de Graduação em enfermagem devidamente matriculados no $6^{\circ}$ e $7^{\circ}$ período. A amostra foi composta por 17 acadêmicos do $6^{\circ}$ e $7^{\circ}$ períodos matutinos e noturnos, de forma aleatória por conveniência.

Foram incluídos na pesquisa os acadêmicos regularmente matriculados no $6^{\circ} \mathrm{e} 7^{\circ}$ períodos matutinos e noturnos e que concordarem em participar do estudo através da assinatura do Termo de Consentimento Livre e Esclarecido (TCLE). Foram excluídos na pesquisa os acadêmicos que não estiverem matriculados nos períodos da coleta de dados no semestre letivo e os que faltaram no dia da coleta dos dados.

Realização de contato com os gestores do Centro Universitário Estácio do Recife para a possibilidade de realização da pesquisa. Apresentação do projeto ao colegiado do curso de Graduação de Enfermagem e entrega da solicitação carta de anuência. Explicação da metodologia de pesquisa aos participantes e entrega dos Termos de Consentimento Livre e Esclarecido (TCLE).

O Instrumento utilizado foi o modelo WHOQOL-Bref de Qualidade de Vida dos profissionais de enfermagem. O modelo WHOQOL-Bref é constituído de 26 perguntas sendo a pergunta de número (1 e 2) sobre qualidade de vida geral; as respostas seguem uma escala de Likert ( 1 a 5 ) quanto maior a porcentagem melhor a qualidade de vida. Fora essas duas questões ( 1 e 2 ) o instrumento tem 24 facetas as quais compõem 4 domínios que são Físico, Psicológico, Relações Sociais e Meio Ambiente. Neste instrumento é necessário também recodificar o valor das questões $(1=5)(2=4)(3=3)(4=2)(5=1)$. As perguntas 1 e 2 deverão conter a seguinte forma: Percepção de qualidade de vida resultados em média de (1 a 5); Satisfação com a saúde (resultados em média de (1 a 5) Cada faceta é só somar os valores da entrevista de (1 a 5 ) e dividir pelo número de participantes, fazer me media onde o resultado vai ser de 1 até 5.

\section{Análise dos dados}

Resultados estão apresentados em forma de tabelas e gráficos construídos através de estatísticas descritivas, frequência, potencial e média. Foi verificado o nível de concordância para cada um dos itens e para o conjunto de itens de cada área de conteúdo. Para o conjunto total de itens do instrumento a concordância foi calculada pelo método estatístico Kappa. O Coeficiente de Kappa de concordância é a razão da proporção de vezes que os indivíduos concordam (corrigido por concordância devido ao acaso) com a proporção máxima de vezes que os indivíduos poderiam concordar (corrigida por concordância devido ao acaso) (Siegeli \& Castellan, 2006). Esta pesquisa foi realizada apenas após a aprovação do projeto pelo comitê de ética em pesquisa CEP: 50.720-635do centro universitário Estácio do Recife foi solicitada a anuência da instituição sede da coleta de dados antes de ser preenchido o questionário.

A confidencialidade e privacidade dos participantes foram estreitamente protegidas durante toda a pesquisa e após a mesma.

Os resultados gerais obtidos foram utilizados apenas e exclusivamente para alcançar os objetivos do trabalho, incluindo sua publicação na literatura cientifica especializada e apresentação em eventos científicos além do retorno a instituição, com o objetivo subsidiar as ações de planejamento de saúde.

A pesquisa cumpriu a resolução 466/2012 do Conselho Nacional de Saúde que dispõe sobre pesquisa com seres humanos, além da resolução Cofen 311/2007 que regulamenta o código de ética dos profissionais de enfermagem (Cofen, 2007; Brasil, 2017). Os riscos estão ligados a algum constrangimento que o universitário poderá ter em responder ao questionário. Mesmo assim essa possibilidade é pequena de forma que esta pesquisa conferiu um grau de risco mínimo. Divulgar estudo que fortaleça a enfermagem na sua responsabilidade, contudo no âmbito social. Os benefícios prever que através deste estudo podemos juntamente com a instituição, elaborar estratégias educativas que venham a melhorar a qualidade devida dos profissionais de enfermagem que conciliam trabalho e estudo durante o decorrer da formação. 


\section{Resultados e discussão}

Nesse tópico foram apresentados os resultados da pesquisa em forma de tabelas seguidos de analise descritiva dos dados. A amostra do estudo foi composta por 17 técnicos de enfermagem que trabalham e estudam, todos responderam os questionários e assinaram o Termo de Consentimento Livre e Esclarecido (TCLE).

Segundo a Organização Mundial da Saúde, a Qualidade de vida pode ser entendida como uma percepção do indivíduo acerca de sua posição na vida, assim como no contexto cultural e no sistema de valores nos quais se encontra inserido e em relação a seus objetivos, expectativas, padrões e preocupações de vida. Para a maioria da população, ter qualidade de vida em si tem um significado mais importante que a preocupação com o tempo que cada indivíduo irá viver. Assim, surge o conceito de qualidade de vida relacionada à saúde, que representa um fator pertinente ao contexto do status de saúde, ou variação do impacto que a doença provoca na vida do indivíduo, evidenciado por ele mesmo (Pereira, Teixeira, \& Santos 2012). A tabela 1 representa os dados sócios demográficos da amostra do estudo.

Tabela 1. Distribuição da amostra quanto ao gênero, faixa etária, Número de vínculos empregatícios, e tipos de vínculos empregatícios, Recife-PE, 2017.

\begin{tabular}{|c|c|c|}
\hline Variáveis & $\mathbf{F}$ & $\%$ \\
\hline \multicolumn{3}{|l|}{ Gênero } \\
\hline Masculino & 3 & $17,65 \%$ \\
\hline Feminino & 14 & $82,35 \%$ \\
\hline Total & 17 & $100 \%$ \\
\hline \multicolumn{3}{|l|}{ Faixa Etária } \\
\hline 26 a 31 anos & 4 & $23,53 \%$ \\
\hline 32 a 37 anos & 7 & $41,18 \%$ \\
\hline 44 a 49 anos & 4 & $23,53 \%$ \\
\hline 50 anos & 2 & $11,76 \%$ \\
\hline Total & 17 & $100 \%$ \\
\hline \multicolumn{3}{|c|}{ Número de Vínculos Empregatícios } \\
\hline 1 & 11 & $64,71 \%$ \\
\hline 2 & 5 & $29,41 \%$ \\
\hline 3 & 1 & $5,88 \%$ \\
\hline Total & 17 & $100 \%$ \\
\hline \multicolumn{3}{|c|}{ Tipo de Vínculo Empregatício } \\
\hline Público & 8 & $47,06 \%$ \\
\hline Privado & 7 & $41,18 \%$ \\
\hline Público + privado & 2 & $11,76 \%$ \\
\hline Total & 17 & $100 \%$ \\
\hline Total & 17 & $100 \%$ \\
\hline
\end{tabular}

De acordo com os dados apresentados na tabela 1, 82,53\% da amostra são do gênero feminino, com 41,18\% na faixa etária entre 32 a 37 anos, 64,71\% tem um vínculo empregatício sendo 47,06\% no Serviço Público e 41,18\% no Serviço Privado. O que se tem observado é que há um crescimento significativo do setor privado nas últimas décadas, comparando-se ao setor público, o que vai contra os princípios de que os serviços privados seriam um complemento ao SUS. Há uma tendência à privatização do sistema de saúde brasileiro, com baixo financiamento público destinado à saúde, o que dificulta a universalização do acesso ao serviço de Saúde (Ocké-Reis, 2012).

De acordo com os dados apresentados na tabela $2,82,35 \%$ da amostra refere ter uma jornada de trabalho semanal 40 horas; $58,82 \%$ em regime de trabalho de $12 \mathrm{~h} \times 36 \mathrm{~h} ; 76,47 \%$ tem renda familiar de 1,5 a 2,0 salários mínimos e $58,82 \%$ estão cursando o $7^{\circ}$ período noturno.

As condições de trabalho da equipe de enfermagem, principalmente nos hospitais, têm sido consideradas impróprias no que concerne às especificidades do ambiente gerador de riscos à saúde. A remuneração inadequada, o acumulo de escalas de serviço, o aumento da jornada de trabalho, as características tensiógenas 
dos serviços de saúde (tanto pela natureza do cuidado prestado às pessoas em situações de risco quanto pela divisão social do trabalho), a hierarquia presente na equipe de saúde e o desprestígio social, entre outros fatores, associam-se às condições de trabalho (Schmoelleret al., 2011). A baixa remuneração e a falta de perspectiva na carreira colaboram para a reduzida satisfação com o componente status profissional, visto que 1,5 a 2,0 salários mínimos são muito pouco para tanta responsabilidade que o profissional exerce em sua vida como: casa, filhos e outras necessidades envolvidas.

Tabela 2. Distribuição da amostra quanto a Jornada de trabalho Semanal, Regime de trabalho, Renda Familiar, Período Acadêmico, Recife-PE, 2017.

\begin{tabular}{|c|c|c|}
\hline Variáveis & $\mathbf{F}$ & $\%$ \\
\hline \multicolumn{3}{|l|}{ Jornada de Trabalho Semanal } \\
\hline 30 horas & 2 & $11,76 \%$ \\
\hline 40 horas & 14 & $82,35 \%$ \\
\hline 30 horas +40 horas & 1 & $5,88 \%$ \\
\hline Total & 17 & $100 \%$ \\
\hline \multicolumn{3}{|l|}{ Regime de trabalho } \\
\hline $12 \times 36$ & 10 & $58,82 \%$ \\
\hline $12 \times 60$ & 4 & $23,53 \%$ \\
\hline $12 \times 36+12 \times 60$ & 1 & $5,88 \%$ \\
\hline $12 \times 36+24 \times 120$ & 1 & $5,88 \%$ \\
\hline $12 \times 60+24 \times 120$ & 1 & $5,88 \%$ \\
\hline Total & 17 & $100 \%$ \\
\hline \multicolumn{3}{|l|}{ Renda Familiar } \\
\hline Até 1 salário mínimo & 2 & $11,76 \%$ \\
\hline Até 1,5 salários mínimos & 7 & $41,18 \%$ \\
\hline Até 2 salários mínimos & 6 & $35,29 \%$ \\
\hline Mais de 2 salários mínimos & 2 & $11,76 \%$ \\
\hline Total & 17 & $100 \%$ \\
\hline \multicolumn{3}{|l|}{ Período Acadêmico } \\
\hline $6^{\circ}$ Manhã & 1 & $5,88 \%$ \\
\hline $6^{\circ}$ Noite & 4 & $23,53 \%$ \\
\hline $7^{\circ}$ Manhã & 2 & $11,76 \%$ \\
\hline $7^{\circ}$ Noite & 10 & 58,82 \\
\hline Total & 17 & $100 \%$ \\
\hline Total & 17 & $100 \%$ \\
\hline
\end{tabular}

O trabalhar e estudar são atividades que frequentemente fazem parte da vida dos indivíduos, principalmente os técnicos de enfermagem que buscam ingressar na educação superior nos dias de hoje, visando uma melhoria na condição financeira e realização profissional (Maier \& Mattos, 2016).

De acordo com os dados apresentados na tabela 3, 94,12\% da amostra tem carga horária de aula semanal de 10 a 15 horas; 88,24\% estudam de 03 a 04 dias de aulas semanais; $41,18 \%$ têm dois filhos; $47,06 \%$ são solteiros e $70,59 \%$ residem na capital.

As exigências atuais estão fortemente marcadas pelo crescimento da produtividade e inserção de novas tecnologias, situação que pode estar associada à busca pela qualificação profissional. Com isso, profissionais do nível médio da enfermagem estão buscando a graduação como um meio decrescimento pessoal, profissional e de conhecimentos. A trajetória acadêmica é uma etapa que se caracteriza por momentos promotores desprazer e de desprazer, considerando-se que é um espaço para aquisição de novos amigos, de novas esperanças, mas também de exigências cognitivas implicadas neste contexto, o que, por si só, pode interferir no curso da qualidade de vida do aluno, seguir apresentaremos os resultados da coleta de dados relacionados à qualidade de vida dos profissionais de enfermagem, foi utilizado um instrumento estruturado de avaliação, o WHOQOL-ABREVIADO ou BREF, elaborado pela Organização Mundial de Saúde /2000. 
Tabela 3. Distribuição da amostra quanto a carga horária de aula semanal, dias de aulas semanais, número de filhos, estado civil, logradouro. Recife-PE, 2017.

\begin{tabular}{|c|c|c|}
\hline Variáveis & $\mathbf{F}$ & $\%$ \\
\hline \multicolumn{3}{|c|}{ Carga horária de aula semanal } \\
\hline 10 horas & 8 & $47,06 \%$ \\
\hline 15 horas & 8 & $47,06 \%$ \\
\hline 20 horas & 1 & $5,88 \%$ \\
\hline Total & 17 & $100 \%$ \\
\hline \multicolumn{3}{|c|}{ Dias de aulas semanais } \\
\hline 02 & 2 & $11,76 \%$ \\
\hline 03 & 7 & $41,18 \%$ \\
\hline 04 & 8 & $47,06 \%$ \\
\hline Total & 17 & $100 \%$ \\
\hline \multicolumn{3}{|c|}{ Número de Filhos } \\
\hline 1 & 3 & $17,65 \%$ \\
\hline 2 & 7 & $41,18 \%$ \\
\hline 3 & 2 & $11,76 \%$ \\
\hline Mais de 3 & 5 & $29,41 \%$ \\
\hline Total & 17 & $100 \%$ \\
\hline \multicolumn{3}{|l|}{ Estado Civil } \\
\hline Solteiro & 8 & $47,06 \%$ \\
\hline Casado & 6 & $35,29 \%$ \\
\hline Separado (a) & 3 & $17,65 \%$ \\
\hline Total & 17 & $100 \%$ \\
\hline \multicolumn{3}{|l|}{ Logradouro } \\
\hline Capital & 12 & $70,59 \%$ \\
\hline Interior & 5 & $29,41 \%$ \\
\hline Total & 17 & $100 \%$ \\
\hline Total & 17 & $100 \%$ \\
\hline
\end{tabular}

Tabela 4. Distribuição da amostra de estudo quando a Avaliação da Qualidade de Vida e Satisfação com a Saúde, Recife-PE, 2017.

\begin{tabular}{ccc}
\hline Variáveis & F & \% \\
\hline Avaliação da qualidade de vida & & \\
Insatisfeito & 1 & $5,88 \%$ \\
Regular & 5 & $29,41 \%$ \\
Satisfeito & 11 & $64,70 \%$ \\
Total & 17 & $100 \%$ \\
\hline Satisfação com a saúde & & \\
Muito pouco & 1 & $5,90 \%$ \\
Regular & 7 & $41,17 \%$ \\
Extremamente & 9 & $52,94 \%$ \\
Total & 17 & $100 \%$ \\
Total & 17 & $100 \%$ \\
\hline
\end{tabular}

De acordo com os dados apresentados na tabela 4, 64,70\% da amostra estão satisfeitos de acordo com a sua qualidade de vida e 52,94\% estão extremamente satisfeitos com sua saúde. A qualidade de vida dos profissionais de enfermagem no âmbito laboral depende da comunicação e das relações interpessoais como também de um ambiente físico favorável. Isto por que a qualidade dos mesmos esta interligada a qualidade dos meios que usam para o desenvolver das suas atividades e as condições de trabalho. Evidencia-se também, que a qualidade de vida desses profissionais é complexa, pois além da saúde física deve-se cuidar da parte emocional, social e principalmente a psicológica (Schmoelleret al., 2011). 
A satisfação acomete quando algo desejado ocorre ou quando o profissional se sente preparado para desenvolver as atividades laborais, é de extrema importância que os profissionais de enfermagem estejam em condições emocionais estruturadas. Mais isso também influenciado pelo ambiente seguro, harmônico para melhor efetivar a assistência de enfermagem (Schmoelleret al., 2011).

Tabela 5. Distribuição da amostra quanto ao Domínio Físico, Recife-PE, 2017.

\begin{tabular}{ccc}
\hline Variáveis & F & \% \\
\hline Relação a dor física & & \\
Muito pouco & 7 & $41,17 \%$ \\
Regular & 9 & $52,94 \%$ \\
Bastante & 1 & $5,88 \%$ \\
Total & 17 & $100 \%$ \\
\hline Tratamento médico para sua vida diária & & \\
Muito pouco & 9 & $52,94 \%$ \\
Regular & 6 & $35,29 \%$ \\
Bastante & 2 & $11,76 \%$ \\
Total & 17 & $100 \%$ \\
\hline Capacidade de energia para seu dia-a-dia & & \\
Muito pouco & 1 & $5,88 \%$ \\
Regular & 5 & $29,41 \%$ \\
Completamente & 11 & $64,70 \%$ \\
Total & 17 & $100 \%$ \\
\hline Capacidade de locomoção & & \\
Muito Bom & 2 & $17,76 \%$ \\
Regular & 10 & $58,82 \%$ \\
Ruim & 5 & $29,41 \%$ \\
Total & 17 & $100 \%$ \\
\hline Total & 17 & $100 \%$ \\
\hline
\end{tabular}

De acordo com os dados apresentados na tabela 5, 52,94\% encontra-se regular em relação a dor física; $52,94 \%$ afirmam ter muito pouco tratamento médico para sua vida diária; $64,70 \%$ tem completamente capacidade de energia no seu dia a dia e 58,82 \% encontra-se regular com sua capacidade de locomoção. Os profissionais de enfermagem devem estar atentos a prevenção de agravos a sua saúde como lombalgia e sintomas osteomusculares em decorrência da prestação da assistência aos seus pacientes, eles se esquecem de cuidar de suas próprias vidas (Silva et al., 2016).

Tabela 6. Distribuição da amostra quanto ao Domínio Físico, Recife-PE, 2017.

\begin{tabular}{ccc}
\hline Variáveis & F & \% \\
\hline Satisfação com o sono & 6 & $35,29 \%$ \\
Insatisfeito & 8 & $47,05 \%$ \\
Regular & 3 & $17,64 \%$ \\
Satisfeito & 17 & $100 \%$ \\
Total & & \\
\hline Satisfação com o desempenho das atividades diárias & 3 & $17,64 \%$ \\
Insatisfeito & 7 & $41,17 \%$ \\
Regular & 7 & $41,17 \%$ \\
Satisfeito & 17 & $100 \%$ \\
Total & & \\
Capacidade para o trabalho & 1 & $5,88 \%$ \\
Insatisfeito & 4 & $23,53 \%$ \\
Regular & 12 & $70,58 \%$ \\
Satisfeito & 17 & $100 \%$ \\
\hline Total & 17 & $100 \%$ \\
\hline
\end{tabular}


De acordo com os dados apresentados na tabela 6, 47,05\% tem satisfação do sono regular; $82,34 \%$ estão regulares e satisfeitos com o desempenho das atividades diárias e 70,58\% estão satisfeitos com a capacidade para o trabalho. Em se tratando das principais dificuldades encontradas pelos acadêmicos trabalhadores, a sonolência diurna é prevalente. A fadiga, segunda dificuldade citada, vem a ser uma consequência da falta de tempo para dormir. Em decorrência destes fatores, manter a concentração durante o período de aula e estágios pode configurar-se como uma dificuldade aos sujeitos e prejudicar o aprendizado (Silva et al., 2016).

A satisfação é um fator que beneficia a motivação e a produtividade dos profissionais de enfermagem, incentivando a dedicação ao trabalho; e desta forma melhorando a qualidade do serviço. A capacidade para o trabalho é uma situação resultante da associação entre o ambiente e o estilo de vida do servidor, sendo motivado por fatores como características sociodemográficas, estilo de vida adequado (Silva et al., 2016).

Tabela 7. Distribuição da amostra quanto ao Domínio Psicológico, Recife-PE, 2017.

\begin{tabular}{|c|c|c|}
\hline Variáveis & $\mathbf{F}$ & $\%$ \\
\hline \multicolumn{3}{|c|}{ Aproveitamento da vida } \\
\hline Muito pouco & 5 & $29,41 \%$ \\
\hline Regular & 2 & $11,76 \%$ \\
\hline Bastante & 10 & $58,82 \%$ \\
\hline Total & 17 & $100 \%$ \\
\hline \multicolumn{3}{|c|}{ Relação ao sentido da vida } \\
\hline Muito pouco & 1 & $5,88 \%$ \\
\hline Regular & 3 & $17,64 \%$ \\
\hline Bastante & 13 & $76,47 \%$ \\
\hline Total & 17 & $100 \%$ \\
\hline \multicolumn{3}{|c|}{ Capacidade de concentração } \\
\hline Muito pouco & 2 & $11,76 \%$ \\
\hline Regular & 11 & $64,70 \%$ \\
\hline Bastante & 4 & $23,52 \%$ \\
\hline Total & 17 & $100 \%$ \\
\hline \multicolumn{3}{|c|}{ Aceitação da aparência física } \\
\hline Muito pouco & 3 & $17,64 \%$ \\
\hline Regular & 3 & $17,64 \%$ \\
\hline Completamente & 11 & $64,70 \%$ \\
\hline Total & 17 & $100 \%$ \\
\hline \multicolumn{3}{|l|}{ Satisfação pessoal } \\
\hline Insatisfeito & 2 & $11,76 \%$ \\
\hline Regular & 6 & $35,29 \%$ \\
\hline Satisfeito & 9 & $52,94 \%$ \\
\hline Total & 17 & $100 \%$ \\
\hline \multicolumn{3}{|c|}{ Sintomas de psiquismo } \\
\hline Nunca & 13 & $76,47 \%$ \\
\hline Frequentemente & 4 & $23,59 \%$ \\
\hline Total & 17 & $100 \%$ \\
\hline Total & 17 & $100 \%$ \\
\hline
\end{tabular}

De acordo com os dados apresentados na tabela 7, 58,82\% referem aproveitar bastante a vida; 76,47\% dizem que sua vida tem bastante sentido; $64,70 \%$ relatam que sua capacidade de concentração é regular; 64,70\% têm aceitação da sua aparência física; 52,94\% estão satisfeitos com as relações pessoais e 76,47\% referem nunca ter apresentado sintomas psíquicos.

Inseridos num mundo globalizado onde nada para, ocorre aumento da carga de trabalho, redução do número de tempo para se fazer o que gostaria, pressões por resultados e mudanças constantes no mercado; é comum ouvir pessoas falando que as horas do dia não são suficientes, a empresa não entende suas necessidades, as famílias reclamam da falta de convívio, sem falar nos problemas de saúdes corriqueiros no início do séc. XXI, fazendo com que muitos profissionais não possua tempo suficiente para aproveitar sua vida de maneira efetiva causando-lhe sérios problemas na vida pessoal e laboral (Amaral et al., 2015).

Alterações no decorrer da vida são consideradas estressoras, principalmente o aumento dos afazeres cotidianos. Tantas atribuições dos alunos implicam negativamente na execução de suas atividades, assim 
como no sono, na qualidade de vida e na ansiedade de maneira que muitos deles sofrem retração, e sua capacidade de concentração é diminuída na maioria das vezes e é manifestada através de desmotivação para o cumprimento dos seus compromissos acadêmicos (Silva et al., 2016).

A aparência física tem um papel primordial na formação e no desenvolvimento dos estereótipos, dado que é a forma mais simples de distinguir e homogeneizar os membros do grupo-alvo. A boa imagem pessoal cada vez mais abre vantagens nas relações sociais e humanas mesmo que, o que mais importa é a essência, na realidade a aparência conta muito e entre apercepção da aparência a avaliação da essência decorre de um intervalo de tempo que fornece beleza sobre o que constrói ou destrói a estética.

Os profissionais de enfermagem estão sujeitos a alterações psíquicas, seguidos da incidência de transtornos mentais e comportamentais, caracterizados principalmente pela presença de episódios depressivos. Apontam também para a alta prevalência de sinais e sintomas que predispõem os trabalhadores de saúde. Todavia, percebe-se que o modo como as queixas psíquicas são abordadas, necessita de uma atenção mais específica, haja vista a possibilidade de identificação e acompanhamento de quadros com potenciais de complicação das funções mentais (Amaral, 2017).

Tabela 8. Distribuição da amostra quanto ao Domínio Relações Sociais, Recife-PE, 2017.

\begin{tabular}{ccc}
\hline Variáveis & F & \% \\
\hline Satisfação com relações pessoais & & \\
Insatisfeito & 1 & $5,88 \%$ \\
Regular & 7 & $41,17 \%$ \\
Satisfeito & 9 & $52,94 \%$ \\
Total & 17 & $100 \%$ \\
\hline Vida sexual & & \\
Insatisfeito & 8 & $40,05 \%$ \\
Regular & 4 & $23,52 \%$ \\
Satisfeito & 5 & $29,41 \%$ \\
Total & 17 & $100 \%$ \\
\hline Apoio de amigos & & \\
Insatisfeito & 10 & $58,82 \%$ \\
Regular & 3 & $16,64 \%$ \\
Satisfeito & 4 & $23,52 \%$ \\
Total & 17 & $100 \%$ \\
\hline Total & 17 & $100 \%$ \\
\hline
\end{tabular}

De acordo com os dados apresentados na tabela 8, 52,94\% estão satisfeitos com suas relações pessoais; cerca de $40,05 \%$ estão insatisfeitos com sua vida sexual e com relação ao apoio de amigos 58,82\% encontramse insatisfeitos.

As relações pessoais se estabelecem a partir de um processo consigo mesmo é de extrema importância que os profissionais de enfermagem possuam essa satisfação além do relacionamento intrapessoal que cria vínculos não só com os profissionais da sua área mais com toda equipe multiprofissional e com os demais profissionais em outras áreas; As relações interpessoais são importantes também pois se estabelecem a partir de um processo de interação entre os membros de uma mesma equipe, criando-se vínculos profissionais, uma condição relacional entre trabalhadores, a fim de executarem uma ação coletiva, e alcançarem um objetivo em comum" (Amaral, 2017).

Praticar sexo com satisfação faz parte dos quatro pilares de qualidade de vida que são: trabalho, família, lazer e sexo. Não há um consenso entre os teóricos sobre a definição da satisfação sexual. A satisfação pode ser entendida como uma maneira na qual os indivíduos alcancem prazer praticando sexo da maneira que gostam, sem prejudicar a eles mesmos, aos parceiros e a terceiros, as consequências da falta de diálogo entre os parceiros Além da falta de conversa, um dos aspectos que colabora para a insatisfação sexual.

De acordo com os dados apresentados na tabela 9, 82,34\% afirmam ter segurança de vida; em relação ao seu ambiente físico saudável cerca de 58,82\% dizem que seu ambiente estar entre regular e extremamente; $64,70 \%$ da amostra refere ter muito pouco dinheiro para suas necessidades diárias e $54,94 \%$ referem estar regular em relação a informações diárias (Silva et al., 2016). 
A abordagem da segurança, saúde e qualidade de vida tem se mostrado cada vez mais emergente e de grande importância na atualidade coorporativa. O conceito tem sido utilizado como forte indicador do grau de satisfação das pessoas que desempenham um trabalho. É importante disponibilizar aos trabalhadores ferramentas que possam por educar, treinar e proporcionar a eles o equilíbrio dos hábitos que interferem na sua segurança, saúde e qualidade de vida, Atuar permanentemente para controlar e minimizar os riscos associados aos seus processos de trabalho, produtos e serviços, e assegurar a integridade e o bem-estar físico e mental das pessoas com as quais se relaciona, em ambientes que estimulem a cooperação, a coesão, a difusão do conhecimento e o desenvolvimento profissional e humano (Acioli Neto, 2013).

Tabela 9. Distribuição da amostra quanto ao Domínio Meio Ambiente, Recife-PE, 2017.

\begin{tabular}{ccc}
\hline Variáveis & F & \% \\
\hline Segurança de vida diária & & $17,64 \%$ \\
Muito pouco & 3 & $41,17 \%$ \\
Regular & 7 & $41,17 \%$ \\
Extremamente & 7 & $100 \%$ \\
Total & 17 & \\
Ambiente físico saudável & & $41,17 \%$ \\
Muito pouco & 7 & $29,41 \%$ \\
Regular & 5 & $29,41 \%$ \\
Bastante & 5 & $100 \%$ \\
Total & 17 & \\
\hline Dinheiro suficiente para as necessidades & & $64,70 \%$ \\
Muito pouco & 11 & $25,72 \%$ \\
Regular & 4 & $11,76 \%$ \\
Completamente & 2 & $100 \%$ \\
Total & 17 & \\
\hline Muito pouco & & $17,64 \%$ \\
Regular & 3 & $54,94 \%$ \\
Bastante & 9 & $29,41 \%$ \\
Total & 5 & $100 \%$ \\
\hline Total & 17 & $100 \%$ \\
\hline
\end{tabular}

Tabela 10. Distribuição da amostra quanto ao Domínio Meio Ambiente, Recife-PE, 2017.

\begin{tabular}{|c|c|c|}
\hline Variáveis & $\mathbf{F}$ & $\%$ \\
\hline \multicolumn{3}{|c|}{ Oportunidade de atividade de lazer } \\
\hline Muito pouco & 8 & $47,05 \%$ \\
\hline Regular & 7 & $41,17 \%$ \\
\hline Bastante & 2 & $11,76 \%$ \\
\hline Total & 17 & $100 \%$ \\
\hline \multicolumn{3}{|c|}{ Condições de moradia } \\
\hline Insatisfeito & 1 & $5,88 \%$ \\
\hline Regular & 4 & $23,52 \%$ \\
\hline Satisfeito & 12 & $70,58 \%$ \\
\hline Total & 17 & $100 \%$ \\
\hline \multicolumn{3}{|c|}{ Acesso aos serviços de saúde } \\
\hline Insatisfeito & 1 & $5,88 \%$ \\
\hline Regular & 10 & $58,82 \%$ \\
\hline Satisfeito & 6 & $35,29 \%$ \\
\hline Total & 17 & $100 \%$ \\
\hline \multicolumn{3}{|c|}{ Meio de transporte } \\
\hline Insatisfeito & 5 & $29,41 \%$ \\
\hline Regular & 7 & $41,17 \%$ \\
\hline Satisfeito & 5 & $29,41 \%$ \\
\hline Total & 17 & $100 \%$ \\
\hline Total & 17 & $100 \%$ \\
\hline
\end{tabular}


De acordo com os dados apresentados na tabela 10, 47,05\% afirmam ter muito pouco oportunidades de lazer; 70,58\% estão satisfeitos com sua condição de moradia; 58,82\% referem ter acesso regular aos serviços de saúde e quanto aos meios de transporte $41,17 \%$ afirmam ser regular o acesso.

A maioria dos profissionais de enfermagem permuta suas oportunidades de lazer, por escalas extras comprovando a necessidade de melhorias da condição financeira que faz diferença na vida pessoal e ambiental (Amaral, 2017).

\section{Considerações finais}

Tendo em conta o objetivo deste estudo, constatou-se à caracterização social que a maioria dos profissionais de enfermagem (técnicos) eram do sexo feminino, a média da faixa etária era 32 a 37 anos, solteiros, possuíam dois filhos e residiam na capital. A grande maioria da amostra deste estudo eram do $7^{\circ}$ período noturno, estudavam de 03 a 04 dias, tendo assim uma carga horária entre 10 a 15 horas semanais. Tinham apenas 01 vínculo empregatício, tendo jornada de trabalho de 40 horas semanais, caracterizada pelo regime de $12 \times 36$ horas de trabalho.

Com relação aos resultados do estudo em questão, os dados foram analisados pelo instrumento WHOQUOL-Bref, que proporcionou através dos resultados, respostas significantes como: satisfação com sua qualidade de vida, sua saúde, suas condições de moradia e sem esquecer-se de sua aparência física e também com suas relações pessoais; completa capacidade de energia para o desempenho de suas atividades e excelente aproveitamento da vida;

Em contrapartida do que foi exposto, a pesquisa apresenta fatores preocupantes para a qualidade de vida destas pessoas, relacionados a: dor física; falta de tratamento médico; de pouca qualidade do sono; insatisfação com a vida sexual e com o apoio de amigos; insegurança quanto ao seu ambiente físico e sua segurança de vida diária; com a pouca ou regular oportunidade de atividades para o lazer; aos meios de transporte e dificuldade de acesso aos serviços de saúde.

Mas o ponto mais marcante nesse estudo foi a baixa remuneração ressaltada pelos profissionais da pesquisa, indicando a necessidade de ser revista pelas instituições, pois as pessoas são motivadas para o trabalho por várias razões e uma delas é a valorização financeira pelo que produzem.

Os resultados encontrados demonstram a importância de se implantar estratégias que busquem melhorar ainda mais a qualidade de vida destes profissionais de enfermagem nos âmbitos físico, social, ambiental e psicológico. Neste contexto que a enfermagem mesmo ainda em processo de construção, vem evoluindo de forma gradativa quanto ao processo de melhoria da qualidade de vida. Porém essa melhoria não se deve apenas a esses trabalhadores, mas também de toda a equipe participante do dia a dia dos mesmos, além de autoridades, das instituições empregadoras e órgãos responsáveis pela defesa dos direitos deles.

Espera-se que as considerações agregadas neste artigo possam ser apreciadas como um estímulo para a realização de novas pesquisas nesta área para que assim, haja constantes e crescentes melhorias referentes a este tema abordado.

\section{Referência}

Acioli Neto, A., Araújo, R., Pitangui, A., Menezes, L., França, E., Costa, E., Andrade, F., \& Correia Junior, M. 2013. Qualidade de vida e nível de atividade física de profissionais de saúde de unidades de terapia intensiva. Revista Brasileira de Atividade Física \& Saúde, 18(06), 711-711.

Amaral, J. F. do, Ribeiro, J. P., \& Paixão, D. X. da. 2015. Qualidade de vida no trabalho dos profissionais de enfermagem em ambiente hospitalar: uma revisão integrativa. Espaço Para a Saúde - Revista de Saúde Pública Do Paraná, 16(1), 66.

Barlem, E. L. D., Lunardi, V. L., Lunardi, G. L., Tomaschewski-Barlem, J. G., da Silveira, R. S., \& Dalmolin, G. L. 2013. Sofrimento moral em trabalhadores de enfermagem. Revista Latino-Americana de Enfermagem, 21(SPL), 79-87.

Bordignon, M., \& Monteiro, M. I. 2016. Violence in the workplace in Nursing: consequences overview. Revista Brasileira de Enfermagem, 69(5), 996-999. https://doi.org/10.1590/0034-7167-2015-0133

Carvalho, D. P. de, Rocha, L. P., Barlem, J. G. T., Dias, J. S., \& Schallenberger, C. D. 2017. Cargas de trabalho e a saúde do trabalhados de enfermagem: revisão interativa*. Cogitare Enfermagem, 22(1).

Cavalheiro, G., \& Tolfo, S. da R. 2011. Trabalho e depressão: um estudo com profissionais afastados do ambiente laboral. Psico-USF, 16(2), 241-249. 
Costa, K. N. D. F. M., Costa, T. F. da, Marques, D. R. F., Marques, F., Viana, L. R. de C., Salviano, G. R., \& Oliveira, M. S. de. 2017. Qualidade de vida relacionada à saúde dos profissionais de enfermagem. Revista de Enfermagem UFPE on Line, 11(2), 881-889.

Daubermann, D. C., \& Tonete, V. L. P. 2012. Qualidade de vida no trabalho do enfermeiro da Atenção Básica à Saúde. ACTA Paulista de Enfermagem, 25(2), 277-283.

Fontana, R. T., \& Brigo, L. (2012). Estudar e trabalhar: percepções de técnicos de enfermagem sobre esta escolha. Escola Anna Nery, 16(1), 128-133.

Freire, M. N., \& Costa, E. R. 2016. Qualidade de vida dos profissionais de enfermagem no ambiente de trabalho. Revista Enfermagem Contemporânea, 5(1), 151-158.

Gomes, R. S., \& Coqueiro, J. F. R. 2016. Qualidade de Vida Relacionada à Carga de Trabalho dos Profissionais de Saúde com enfoque nos problemas desencadeados. Id on Line Revista de Psicologia, 10(33), 249-261.

Hipólito, M. C. V., Masson, V. A., Monteiro, M. I., \& Gutierrez, G. L. 201). Quality of working life: assessment of intervention studies. In Revista brasileira de enfermagem (Vol. 70, Issue 1, pp. 189-197).

Leal, D. R., Mattos, G. D. de, \& Fontana, R. T. 2013. Worker with physical disability: weaknesses and disorders self referred. Revista Brasileira de Enfermagem, 66(1), 59-66.

Lima, P. C., Gouveia, M. T. D. O., Sabino, K. C. V., Fernandes, M. A., \& Avelino, F. V. S. D. 2015. Fatores estressores e as estratégias de enfrentamento utilizadas por enfermeiros hospitalares: revisão. Investigación En Enfermería: Imagen y Desarrollo, 17(2), 51-56.

Maciel, M. E. D., \& Oliveira, F. N. de. 2014. Qualidade de vida do profissional técnico de enfermagem: a realidade de um hospital filantrópico em Dourados-MS. Revista Psicologia e Saúde, 6(1), 83-89.

Maier, S. R. de O., \& Mattos, M. De. 2016. O Trabalhar e o Estudar no Contexto Universitário: Uma Abordagem Com Trabalhadores-Estudantes. Saúde (Santa Maria), 42(1), 185.

Marcitelli, C. R. de A. (2011). Qualidade De Vida No Trabalho Dos Profissionais De Saúde. Ensaios e Ciência: Ciências Biológicas, Agrárias e Da Saúde, 15(4), 215-228.

Marconi, M. D. A., \& Lakatos, E. M. 2009. Fundamentos de metodologia científica (5a ed). São Paulo, SP: Atlas.

Mascarenhas, C. H. M., Prado, F. O., Fernandes, M. H., Boery, E. N., \& Sena, E. L. da S. 2013. Qualidade de vida em trabalhadores da área de saúde: uma revisão sistemática. Espaç. Saúde (Online), 14(1/2), 72-81.

Mendonça, S. H. A., \& Araújo, L. S. 2016. Esgotamento profissional e qualidade de vida no trabalho: uma revisão integrativa. Psicologias, 2(0), 1-19.

Moura, I. H. de, Nobre, R. de S., Cortez, R. M. A., Campelo, V., Macêdo, S. F. de, \& Silva, A. R. V. 2016. Quality of life of undergraduate nursing students. Revista Gaucha de Enfermagem, 37(2), e55291.

Oliveira, B. M. de, Mininel, V. A., \& Felli, V. E. A. 2011. Qualidade de vida de graduandos de enfermagem. Revista Brasileira de Enfermagem, 64(1), 130-135.

Pereira, É. F., Teixeira, C. S., \& dos Santos, A. 2012. Qualidade de vida: abordagens, conceitos e avaliação. Revista Brasileira de Educação Física e Esporte, 26(2), 241-250.

Ratochinski, C. M. W., Powlowytsch, P. W. da M., Grzelczak, M. T., Souza, W. C. de, \& Mascarenhas, L. P. G. 2016. O Estresse em Profissionais de Enfermagem: Uma Revisão Sistemática. Revista Brasileira de Ciências Da Saúde, 20(4), 341-346.

Rios, K. A., Barbosa, D. A., \& Belasco, A. G. S. 2010. Avaliação de qualidade de vida e depressão de técnicos e auxiliares de enfermagem. Revista Latino-Americana de Enfermagem, 18(3), 413-420.

Sampaio, J. R. 2012. Qualidade de Vida no Trabalho: Perspectivas e Desafios Atuais Quality of working life: Actual perspectives and challenges. Revista Psicologia: Organização e Trabalho, 12(1), 121-136.

Santana, L. de L., Sarquis, L. M. M., Miranda, F. M. D. A., Kalinke, L. P., Felli, V. E. A., \& Mininel, V. A. 2016. Health indicators of workers of the hospital area. Revista Brasileira de Enfermagem, 69(1), 23-32.

Santana, V. S., Feitosa, A. G., Arruda Guedes, L. B., \& Brito Sales, N. B. 2014. Qualidade de Vida dos Profissionais de saúde em Ambiente Hospitalar . Revista Pesquisa Em Fisioterapia, 4(1), 46.

Santos Fonseca, I. S., Silva, R. B., Silveira, C. M. C., \& Santa Rosa, M. P. R. 2014. Qualidade de vida no trabalho dos profissionais de enfermagem: uma análise da produção científica entre 2000 e 2012. Interfaces Científicas - Saúde e Ambiente, 3(1), 19-28.

Schmidt, D. R. C., Paladini, M., Biato, C., Pais, J. D., \& Oliveira, A. R. 2013. Qualidade de vida no trabalho e burnout em trabalhadores de enfermagem de Unidade de Terapia Intensiva. Revista Brasileira de Enfermagem, 66(1), 13-17. 
Schmoeller, R., Trindade, L. D. L., Neis, M. B., Gelbcke, F. L., \& Pires, D. E. P. D. 2011. Cargas de trabalho e condições de trabalho da enfermagem: revisão integrativa. Revista Gaúcha de Enfermagem, 32(2), 368-377.

Schrader, G., Palagi, S., Padilha, M. A. S., Noguez, P. T., Thofehrn, M. B., \& Dal Pai, D. 2012. Trabalho na Unidade Básica de Saúde: implicações para a qualidade de vida dos enfermeiros. Revista Brasileira de Enfermagem, 65(2), 222-228.

Siegel, S., \& Castellan Jr, N. J. 1975. Estatística não-paramétrica para ciências do comportamento. Artmed Editora.

Silva, A. E., Lima, P. K. M., \& Oliveira, C. 2016. Qualidade de vida dos profissionais de enfermagem nível médio em unidade de terapia intensiva. Revista de Enfermagem Do Centro-Oeste Mineiro, 6(3), 2318-2330.

Silva, A. P. da, \& Queiroz, E. de S. 2011. NBC-Periódico Científico do Núcleo de Biociências O estresse e sua relação com a jornada de trabalho da enfermagem em unidade hospitalar. NBC - Periódico Cientifico Do Núcleo de Biociência , 1(1), 34-50.

Silva, D. dos S. D., Tavares, N. V. da S., Alexandre, A. R. G., Freitas, D. A., Brêda, M. Z., de Albuquerque, M. C. dos S., \& de Melo Neto, V. L. 2015. Depressão e risco de suicídio entre profissionais de Enfermagem: revisão integrativa. Revista Da Escola de Enfermagem Da USP, 49(6), 1023-1031.

Ueno, L. G. S., Bobroff, M. C. C., Martins, J. T., Bueno, R. C., Machado, R., Linares, P. G., \& Gaspar, S. G. 2017. Estresse ocupacional: estressores referidos pela equipe de enfermagem. Revista de Enfermagem UFPE on Line, 11(4), 1638.

Viana, V. C. D. A., Santos, F. P. dos A., Nery, A. A., Oliveira, J. da S., \& Almeida, C. L. S. 2016. Condições de trabalho dos enfermeiros na estratégia saúde da família: revisão integrativa. Revista de Enfermagem UFPE Online, 10(7), 2696-2707.

Zavala, M. O. Q., Klinj, T. P., \& Carrillo, K. L. S. 2016. Qualidade de vida no trabalho do pessoal de enfermagem de instituições públicas de saúde . Revista Latino Americana De Enfermagem , 24, e2713.

\section{Minicurrículo}

Tássia Rayane de Arruda. Graduada em Enfermagem pela Universidade Estácio do Recife (2018); pósgraduada em Enfermagem Psiquiátrica e Saúde Mental (2021), pós-graduanda em Enfermagem em Urgência e Emergência e Enfermagem em UTI (2021).

Wagner Ferreira dos Santos. Técnico em enfermagem; Bacharelado em enfermagem, FAPI (Faculdade do Piauí). Treinamento em atendimento pré-hospitalar promovido pelo núcleo de educação permanente do SAMU Recife-PE, urgências respiratórias e ventilação mecânica atendimento pré-hospitalar ofertado pela Emergência, Enfermagem Obstétrica, Atendimento pré-hospitalar a queimados.

Natália Izabel Domingos de Freitas. Pós-Graduanda em Urgência e Emergência Atendimento Pré Hospitalar, Avaliação e tratamento de feridas Urgência e emergência em Home Care, Administração de Medicações em Home Care, Assistência ao paciente com Piccline e Interpretação e Evolução em prescrição médica Punção Venosa Periférica

Como citar: Arruda, T.R., Santos, W.F., \& Freitas, N.I.D. 2021. Qualidade de vida dos profissionais de enfermagem que conciliam trabalho e estudo em um centro universitário privado na cidade de Recife/PE. Pubsaúde, 6, a155. DOI: https://dx.doi.org/10.31533/pubsaude6.a155

Recebido: 12 abr. 2021.

Revisado e aceito: 22 abr. 2021.

Conflito de interesse: os autores declaram, em relação aos produtos e companhias descritos nesse artigo, não ter interesses associativos, comerciais, de propriedade ou financeiros que representem conflito de interesse.

Licenciamento: Este artigo é publicado na modalidade Acesso Aberto sob a licença Creative Commons Atribuição 4.0 (CC-BY 4.0). 